\title{
RIESZ-MARTIN REPRESENTATION FOR POSITIVE SUPER-POLYHARMONIC FUNCTIONS IN A RIEMANNIAN MANIFOLD
}

\author{
V. ANANDAM AND S. I. OTHMAN
}

Received 19 December 2005; Revised 28 February 2006; Accepted 4 April 2006

Let $u$ be a super-biharmonic function, that is, $\Delta^{2} u \geq 0$, on the unit disc $D$ in the complex plane, satisfying certain conditions. Then it has been shown that $u$ has a representation analogous to the Poisson-Jensen representation for subharmonic functions on $D$. In the same vein, it is shown here that a function $u$ on any Green domain $\Omega$ in a Riemannian manifold satisfying the conditions $(-\Delta)^{i} u \geq 0$ for $0 \leq i \leq m$ has a representation analogous to the Riesz-Martin representation for positive superharmonic functions on $\Omega$.

Copyright (c) 2006 Hindawi Publishing Corporation. All rights reserved.

\section{Introduction}

Let $u$ be a locally Lebesgue integrable function defined on the unit disc $D$ in the complex plane. $u$ is called a super-biharmonic function if $\Delta^{2} u \geq 0$ in the sense of distributions. Abkar and Hedenmalm [1] consider a super-biharmonic function $u$ on $D$, satisfying two conditions which regulate the growth of $u$ near the boundary $\partial D$. These conditions are used to split $u$ into its biharmonic Green potential part and its biharmonic part. Using this decomposition, they show that $u$ can be represented by three measures, one on $D$ and two on the boundary $\partial D$. This comes out as a generalization of the Riesz-Poisson integrals to the super-biharmonic functions on $D$. However, an extension of this representation in the case of the unit ball in $\mathbb{R}^{n}, n>2$ (or to the case of $\Delta^{m} u \geq 0$ with suitable restrictions on $u$ in the unit disc itself) seems complicated.

In this paper, we consider a set of two other conditions on a function $u$ satisfying $\Delta^{2} u \geq$ 0 , namely, $u \geq 0$ and $\Delta u \leq 0$. These conditions are more appropriate as a generalization of the positive superharmonic functions. For, suppose $u$ is a locally Lebesgue integrable function on a bounded domain $\Omega$ in $\mathbb{R}^{n}, n \geq 2$, such that $u \geq 0, \Delta u \leq 0$, and $\Delta^{2} u \geq 0$. Then $u$ can be represented by three positive measures, one on $\Omega$ and two on the Martin boundary of $\Omega$. Interestingly, the method of proof is general enough to be used in the case of $(-\Delta)^{i} u \geq 0,0 \leq i \leq m$, for any integer $m \geq 2$, and any domain $\Omega$ in $\mathbb{R}^{n}$ on which the Green function is defined (in particular on any bounded domain $\Omega$ in $\mathbb{R}^{n}, n \geq 2$ ); actually, it goes through in the case of a Riemannian manifold also. Accordingly, we prove this result in the context of a Riemannian manifold. 


\section{Preliminaries}

Let $R$ be an oriented Riemannian manifold of dimension $\geq 2$, with local coordinates $x=$ $\left(x^{1}, \ldots, x^{n}\right)$ and a $C^{\infty}$-metric tensor $g_{i j}$ such that $g_{i j} x^{i} x^{j}$ is positive definite. Denote the

volume element by $d x=\sqrt{\operatorname{det}\left(g_{i j}\right)} d x^{1}, \ldots, d x^{n}$. Let $\Delta$ be the Laplace-Beltrami operator which, acting on a $C^{2}$-function $f$, gives $\Delta f=\operatorname{div}$ grad $f$. However, we will assume that $\Delta$ is taken in the sense of distributions. Thus, a locally $d x$-integrable function $f$ on an open set $\omega$ in $R$ is said to be superharmonic (resp., harmonic) if $\Delta f \leq 0$ (resp., $\Delta f=0$ ) on $\omega$; a positive superharmonic function $u$ on $\omega$ is called a potential if and only if the greatest harmonic minorant of $u$ on $\omega$ is 0 , (i.e., if $h$ is harmonic on $\omega$ and $h \leq u$, then $h$ should be negative).

For each open set $\omega$ in $R$, let $H(\omega)$ denote the class of $C^{2}$-functions $u$ on $\omega$ such that $\Delta u=0$. If $\omega$ is a domain, $H(\omega)$ has the Harnack property, namely, if $h_{n}$ is an increasing sequence in $H(\omega)$ and if $h=\sup h_{n}$, then $h \in H(\omega)$ or $h \equiv \infty$. We can also solve the Dirichlet problem on any parametric ball. This means that the set of harmonic functions $H(\omega)$ satisfies the axioms 1, 2, 3 of Brelot [7, pages 13-14]. Consequently, we can use the results and the terminology of the Brelot axiomatic potential theory in the context of the Riemannian manifold $R$.

A domain $\Omega$ in $R$ is called a Green domain if the Green function $G(x, y)$ is well defined on $\Omega$. On a Green domain $\Omega$ in $R$, we can construct the Martin compactification $\bar{\Omega}$ of $\Omega$ as in [8, pages 111-115]. Some of the important points to remember here are the following: fix a point $y_{0}$ in a Green domain $\Omega$. If $G(x, y)$ is the Green function on $\Omega$, write $k_{y}(x)=k(x, y)=G(x, y) / G\left(x, y_{0}\right)$ with the convention $k\left(y_{0}, y_{0}\right)=1$. Then there exists only one (metrizable) compactification $\bar{\Omega}$ up to homeomorphism such that

(i) $\Omega$ is dense open in the compact space $\bar{\Omega}$;

(ii) $k_{y}(x), y \in \Omega$, extends as a continuous function of $x$ on $\bar{\Omega}$;

(iii) the family of these extended continuous functions on $\bar{\Omega}$ separates the points $x \in$ $\Delta=\bar{\Omega} \backslash \Omega$.

$\bar{\Omega}$ is called the Martin compactification of $\Omega$ and $\Delta=\bar{\Omega} \backslash \Omega$ is called the Martin boundary. A positive harmonic function $u>0$ is called minimal if and only if for any harmonic function $v, 0 \leq v \leq u$, we should have $v=\alpha u$ for a constant $\alpha, 0 \leq \alpha \leq 1$. It can be proved that every minimal harmonic function $u(y)$ on $\Omega$ is of the form $u\left(y_{0}\right) k(x, y)$ for some $x \in \Delta$, and the points $x \in \Delta$ corresponding to these minimal harmonic functions are called the minimal points of $\Delta$, and the set of minimal points of $\Delta$ is denoted by $\Delta_{1}$, called the minimal boundary.

With these remarks, we can state the Martin representation theorem: for any harmonic function $u \geq 0$ on $\Omega$, there exists a unique Radon measure $\mu \geq 0$ on $\Delta$ with support in the minimal boundary $\Delta_{1} \subset \Delta$ such that $u(y)=\int_{\Delta_{1}} k(x, y) d \mu(x)$.

In the particular case of $R=\mathbb{R}^{n}, n \geq 2$, and $\Omega=B(0,1)$ the unit ball, taking the fixed point $y_{0}$ as the centre 0 , we have the following: the Martin boundary $\Delta=\bar{\Omega} \backslash \Omega$ is homeomorphic to the unit sphere $S$ and $k(x, y)$ is the Poisson kernel; also $\Delta_{1}=\Delta=S$. Then the Martin representation gives the familiar result (see, e.g., Axler et al. [4, page 105]): if $u$ is positive and harmonic on $B$, then there exists a unique positive Borel measure on $S$ such that $u(x)=\int_{S} p(x, y) d \mu(y)$, where $p(x, y), x \in B, y \in S$, is the Poisson kernel. 


\section{Riesz-Martin representation for positive super-biharmonic functions}

Let $\Omega$ be a Green domain in a Riemannian manifold $R$, with the Green function $G(x, y)$ which is a symmetric function and for fixed $y, G_{y}(x)=G(x, y)$ is a potential on $\Omega$; we have also $\Delta G_{y}(x)=-\delta_{y}(x)$, after a normalization.

Definition 3.1. A Green domain $\Omega$ in $R$ called a biharmonic Green domain if for a pair of points $x$ and $y$ in $\Omega, G^{2}(x, y)=\int_{\Omega} G(x, z) G(z, y) d z$ is finite. Then $G^{2}(x, y)$ is called the biharmonic Green function of $\Omega$.

The above definition is given in Sario [10] when $\Omega=R$, a hyperbolic manifold. On an arbitrary hyperbolic Riemannian manifold $R$, the biharmonic Green function may or may not exist. It is shown in [2, Theorem 3.2] that the biharmonic Green function $G^{2}(x, y)$ can be defined on a hyperbolic Riemannian manifold $R$ if and only if there exist two positive potentials $p$ and $q$ on $R$ such that $\Delta q=-p$.

Consequently, any relatively compact domain $\Omega$ in a Riemannian manifold $R$ is a biharmonic Green domain, whether $R$ is hyperbolic or parabolic. Note that if $\Omega$ is a biharmonic Green domain in $R$, then $u(x)=G^{2}(x, y)$ is a potential on $\Omega$, for fixed $y$; and $\Delta u(x)=\Delta_{x} G_{y}^{2}(x)=-G_{y}(x)$ so that $\Delta^{2} u(x)=\delta_{y}(x)$.

Given a Radon measure $\mu \geq 0$ on $\Omega$, if we set $p(x)=\int_{\Omega} G(x, y) d \mu(y)$, then we know that $p \equiv \infty$ or $p(x)$ is a potential such that $\Delta p=-\mu$. Let now $q(x)=\int_{\Omega} G^{2}(x, y) d \mu(y)$ be finite at some point $x_{0} \in \Omega$. Then,

$$
\infty>\int_{\Omega}\left(\int_{\Omega} G\left(x_{0}, z\right) G(z, y) d z\right) d \mu(y)=\int_{\Omega} G\left(x_{0}, z\right)\left[\int_{\Omega} G(z, y) d \mu(y)\right] d z .
$$

Hence $p(z)=\int_{\Omega} G(z, y) d \mu(y) \neq \equiv$, so that $p(z)$ is a potential on $\Omega$, and $q(x)=\int_{\Omega} G(x, z) p(z) d z$, which shows that $q(x)$ is a potential on $\Omega$ and $\Delta q(x)=-p(x)=-\int_{\Omega} G(x, y) d \mu(y)$.

Let $\bar{\Omega}$ be the Martin compactification of $\Omega, \Delta=\bar{\Omega} \backslash \Omega$ the Martin boundary, and $\Delta_{1}$ the minimal boundary $\subset \Delta$. Let $k(x, y)$ be the Martin kernel, $(x, y) \in \bar{\Omega} \times \Omega$.

Notation 3.2. (1) Let $\pi_{2}$ denote the set of positive Radon measures $\mu$ on $\Omega$ such that $q(x)=\int_{\Omega} G^{2}(x, y) d \mu(y)$ is a potential on $\Omega$.

(2) Let $\wedge_{0}$ denote the set of positive Radon measures $v$ on $\Delta$, with supp $v \subset \Delta_{1}$.

(3) Let $\wedge_{1}$ denote the positive Radon measures $v \in \wedge_{0}$ such that $u(x)=\int_{\Omega} G(x$, $y)\left[\int_{\Delta_{1}} k(X, y) d v(X)\right] d y$ is a potential on $\Omega$. In that case, $\Delta u(x)=-\int_{\Delta_{1}} k(X, x) d v(X)$ which is harmonic, so that $u(x)$ is also a biharmonic function on $\Omega$. (Remark that $\wedge_{1}$ can be empty as in the case of $\Omega=\mathbb{R}^{n}$.) If $v \in \wedge_{1}$, we will write $k_{1}(X, x)=\int_{\Omega} G(x, y) k(X, y) d y$ for $X \in \Delta_{1}$, and $x \in \Omega$, so that $u(x)=\int_{\Omega} G(x, y)\left[\int_{\Delta_{1}} k(X, y) d v(X)\right] d y$ can be more elegantly represented as $u(x)=\int_{\Delta_{1}} k_{1}(X, x) d v(X)$.

Lemma 3.3. Let $\mu \geq 0$ be a Radon measure on an open set $\omega$ in a Riemannian manifold $R$, hyperbolic or parabolic. Then there exists a superharmonic function son $\omega$ with $\mu$ as the associated measure in a local Riesz representation.

Proof. The statement means that for every point $x_{0} \in \omega$, there is a neighborhood $\delta$, $x_{0} \in \delta \subset \bar{\delta} \subset \omega$, with the Green function $G^{\delta}(x, y)$ such that $s(x)=\int_{\delta} G^{\delta}(x, y) d \mu(y)+$ (a harmonic function $h(x))$ in $\delta$. 
For the construction of $s$ in $\mathbb{R}^{n}$, we refer to Brelot [6]. A similar method, with the use of an approximation property given in Bagby and Blanchet [5, Theorem 3.10], proves the result in a Riemannian manifold. (For a more general discussion of this result, see [3, Section 2].)

By Definition 3.1, a Green domain $\Omega$ in a Riemannian manifold $R$ (whether hyperbolic or parabolic) is a biharmonic Green domain if and only if $G^{2}(x, y) \not \equiv \infty$ on $\Omega$. Note that $u(x)=G_{y}^{2}(x)>0, \Delta u(x)=G_{y}(x)<0$, and $\Delta^{2} u(x)=\delta_{y}(x) \geq 0$ on $\Omega$. Hence on a biharmonic Green domain $\Omega$, functions $v$ of the type $v>0, \Delta v \leq 0$, and $\Delta^{2} v \geq 0$ exist. The following theorem gives an integral representation for such functions.

Theorem 3.4. Let $\Omega$ be a biharmonic Green domain in a Riemannian manifold $R$ (whether $R$ is hyperbolic or parabolic) and let $v$ be a locally $d x$-integrable function on $\Omega$. Then the following are equivalent.

(a) $v \geq 0, \Delta v \leq 0$, and $\Delta^{2} v \geq 0$ on $\Omega$.

(b) $v(x)=\int_{\Omega} G^{2}(x, y) d \mu(y)+\int_{\Delta_{1}} k_{1}(X, x) d v_{1}(X)+\int_{\Delta_{1}} k(X, x) d v_{0}(X)$ a.e. on $\Omega$, where $\left(\mu, v_{1}, v_{0}\right) \in \pi_{2} \times \wedge_{1} \times \wedge_{0}$ is uniquely determined.

Proof. (b) $\Rightarrow(\mathrm{a})$. Let

$$
u(x)=\int_{\Omega} G^{2}(x, y) d \mu(y)+\int_{\Delta_{1}} k_{1}(X, x) d v_{1}(X)+\int_{\Delta_{1}} k(X, x) d v_{0}(X),
$$

where $\mu \geq 0$ is a Radon measure on $\Omega$, and $v_{0}, v_{1}$ are positive Radon measures on $\Delta_{1}$. Then $u(x)=v(x)$ a.e. on $\Omega$ by the assumption. Hence $u \neq \equiv$.

(i) Let $u_{1}(x)=\int_{\Omega} G^{2}(x, y) d \mu(y)$. Then $u_{1} \geq 0$ is a potential on $\Omega$, such that $\Delta u_{1}(x)=$ $-\int G(x, y) d \mu(y)$ and $\Delta^{2} u_{1}=\mu$.

(ii) Let

$$
u_{2}(x)=\int_{\Delta_{1}} k_{1}(X, x) d v_{1}(X)=\int_{\Omega} G(x, y)\left[\int_{\Delta_{1}} k(X, y) d v_{1}(X)\right] d y
$$

Then $u_{2} \geq 0$ is a potential on $\Omega$, such that $\Delta u_{2}(x)=-\int_{\Delta_{1}} k(X, x) d v_{1}(X)=-h_{1}(x)$, where $h_{1}(x)$ is a positive harmonic function on $\Omega$, so that $\Delta u_{2} \leq 0$ and $\Delta^{2} u_{2} \equiv 0$.

(iii) Let $u_{3}(x)=\int_{\Delta_{1}} k(X, x) d v_{0}(X)$.

Then $u_{3} \geq 0$ is harmonic on $\Omega$, so that $\Delta u_{3} \equiv 0$ and $\Delta^{2} u_{3} \equiv 0$.

Consequently, $u=u_{1}+u_{2}+u_{3} \geq 0$ on $\Omega$ such that $\Delta u \leq 0$ and $\Delta^{2} u \geq 0$ on $\Omega$. Since $u=v$ a.e., the statement (a) is proved.

(a) $\Rightarrow$ (b). Since $\Delta^{2} v \geq 0, \Delta^{2} v=\mu$, where $\mu$ is a positive Radon measure on $\Omega$. Since $\Delta(\Delta v)=\mu, \Delta v$ is a subharmonic function on $\Omega$. Since $\Delta v \leq 0$ by hypothesis, $-\Delta v$ is a positive superharmonic function on $\Omega$. Hence by the Riesz representation theorem,

$$
-\Delta v(x)=\int_{\Omega} G(x, y) d \mu(y)+h(x)
$$

where $h(x)$ is a positive harmonic function on $\Omega$. 
Let us choose (using the lemma above) two superharmonic functions $q(x)$ and $H(x)$ on $\Omega$ such that

$$
\begin{gathered}
\Delta q(x)=-\int_{\Omega} G(x, y) d \mu(y), \\
\Delta H(x)=-h(x) .
\end{gathered}
$$

Then from (3.4),

$$
v(x)=q(x)+H(x)+\left(\text { a harmonic function } h_{1}\right) \quad \text { on } \Omega .
$$

Since $v \geq 0$ on $\Omega, q(x) \geq-H(x)-h_{1}(x)$; that is, $q(x)$ has a subharmonic minorant on $\Omega$. Hence $q(x)$ has the greatest harmonic minorant $h_{2}(x)$ on $\Omega$, and by the Riesz representation theorem,

$$
\begin{aligned}
q(x) & =\int_{\Omega} G(x, y)(-\Delta q(y)) d y+h_{2}(x) \text { on } \Omega \\
& =\int_{\Omega} G(x, z)\left[\int_{\Omega} G(z, y) d \mu(y)\right] d z+h_{2}(x) \\
& =\int_{\Omega} G^{2}(x, y) d \mu(y)+h_{2}(x) .
\end{aligned}
$$

Similarly, dealing with the superharmonic function $H(x)$ and its greatest harmonic minorant $h_{3}(x)$ on $\Omega$, we can write

$$
\begin{aligned}
H(x) & =\int_{\Omega} G(x, y)(-\Delta H(y)) d y+h_{3}(x) \quad \text { on } \Omega \\
& =\int_{\Omega} G(x, y) h(y) d y+h_{3}(x) \\
& =\int_{\Omega} G(x, y)\left(\int_{\Delta_{1}} k(X, y) d v_{1}(X)\right) d y+h_{3}(x),
\end{aligned}
$$

by using the Martin representation for the positive harmonic function $h$ on $\Omega$. Note that $v_{1} \in \wedge_{1}$ and is uniquely determined. Consequently,

$$
H(x)=\int_{\Delta_{1}} k_{1}(X, x) d v_{1}(X)+h_{3}(x)
$$

Now, using (3.6), (3.7), and (3.9), we write

$$
v(x)=\int_{\Omega} G^{2}(x, y) d \mu(y)+\int_{\Delta_{1}} k_{1}(X, x) d v_{1}(X)+h_{0}(x)
$$

where $h_{0}=h_{1}+h_{2}+h_{3}$ is harmonic on $\Omega$.

Now by hypothesis $v \geq 0$, so that

$$
-h_{0}(x) \leq \int_{\Omega} G^{2}(x, y) d \mu(y)+\int_{\Delta_{1}} k_{1}(X, x) d v_{1}(X) \quad \text { on } \Omega .
$$


Now the two terms on the right side are potentials on $\Omega$ and hence their sum also is a potential on $\Omega$. This means that the harmonic function $-h_{0}$ is majorized by a potential on $\Omega$, so that $-h_{0} \leq 0$. Thus $h_{0}$ is a positive harmonic function $\Omega$. Use the Martin representation to conclude that there exists a unique measure $v_{0}$ on the Martin boundary with support in $\Delta_{1}$, such that

$$
h_{0}(x)=\int_{\Delta_{1}} k(X, x) d v_{0}(X)
$$

Thus, from (3.10) and (3.12), we finally arrive at the representation for $v(x)$ on $\Omega$ :

$$
v(x)=\int_{\Omega} G^{2}(x, y) d \mu(y)+\int_{\Delta_{1}} k_{1}(X, x) d v_{1}(X)+\int_{\Delta_{1}} k(X, x) d v_{0}(X),
$$

where $\left(\mu, v_{1}, v_{0}\right) \in \pi_{2} \times \wedge_{1} \times \wedge_{0}$ is uniquely determined.

\section{Representation for positive super-polyharmonic functions}

By induction, we can extend Theorem 3.4 to obtain the Riesz-Martin representation for positive super-polyharmonic functions.

Let $\Omega$ be a Green domain in a Riemannian manifold $R$, with $G(x, y)$ as the Green function of $\Omega$. For an integer $m \geq 2$, we will denote

$$
G^{m}(x, y)=\int G\left(x, z_{m-1}\right) G\left(z_{m-1}, z_{m-2}\right) \cdots G\left(z_{1}, y\right) d z_{1} \cdots d z_{m-1}
$$

and say that a positive Radon measure $\mu$ on $\Omega$ is in $\pi_{m}$ if $u(x)=\int_{\Omega} G^{m}(x, y) d \mu(y) \not \equiv \infty$ on $\Omega$, in which case $u(x)$ is a potential on $\Omega$ and $(-\Delta)^{m} u=\mu$; also $(-\Delta)^{j} u \geq 0$ for $0 \leq j \leq m$. When such a function $u(x)$ exists on $\Omega$, we say that $\Omega$ is an $m$-harmonic Green domain in $R$, whether $R$ is hyperbolic or parabolic.

Let $\bar{\Omega}$ be the Martin compactification of $\Omega$ and let $k(x, y)$ be the Martin kernel. For any $i, 1 \leq i \leq m-1$, let $\wedge_{i}$ denote the set of positive Radon measures $v_{i}$ on $\Delta=\bar{\Omega} \backslash \Omega$ with support in the minimal boundary $\Delta_{1}$, such that

$$
v_{i}(x)=\int G\left(x, z_{i}\right) G\left(z_{i}, z_{i-1}\right) \cdots G\left(z_{2}, z_{1}\right)\left[\int_{\Delta_{1}} k\left(X, z_{1}\right) d v(X)\right] d z_{1} \cdots d z_{i} \neq \equiv \infty .
$$

In that case, $v_{i}(x)$ is a potential on $\Omega,(-\Delta)^{i} v_{i} \equiv 0$; also $(-\Delta)^{j} v_{i} \geq 0$ for $0 \leq j \leq i$. Let us write for $X \in \Delta_{1}$ and $x \in \Omega$,

$$
k_{i}(X, y)=\int G\left(x, z_{i}\right) \cdots G\left(z_{2}, z_{1}\right) k\left(X, z_{1}\right) d z_{1} \cdots d z_{i} .
$$

Then, if $v \in \wedge_{i}, v_{i}(x)=\int_{\Delta_{1}} k_{i}(X, x) d v(X)$ is well defined on $\Omega$ with the above properties.

As before, let $\wedge_{0}$ denote the set of positive Radon measures $v$ on $\Delta$, with support in $\Delta_{1}$.

Then, the proof of Theorem 3.4 can be extended by using the method of induction to arrive at the following result.

Theorem 4.1. Let $\Omega$ be an m-harmonic Green domain in a Riemannian manifold $R$ and let $v$ be a locally $d x$-integrable function on $\Omega$. Let $m \geq 1$ be an integer. Then the following 
are equivalent.

(a) $(-\Delta)^{i} v \geq 0$ on $\Omega$ for $0 \leq i \leq m$.

(b) There exist unique measures $\mu \in \pi_{m}$ and $v_{i} \in \wedge_{i}$ for $0 \leq i \leq m-1$ such that

$$
v(x)=\int_{\Omega} G^{m}(x, y) d \mu(y)+\sum_{i=0}^{m-1} \int_{\Delta_{1}} k_{i}(X, x) d v_{i}(X) \quad \text { a.e. on } \Omega \text {. }
$$

\section{Integral representations in a Riemann surface}

We are not in a position to say that the above integral representation theorems in a Riemannian manifold $R$ are automatically valid in a Riemann surface $S$. For, we have used the Laplace-Beltrami operator $\Delta$ on $R$ to define polyharmonic-superharmonic functions on $R$ and also to obtain some of their properties. But the Laplacian is not invariant under a parametric change in an abstract Riemann surface $S$. Hence there is a problem. We indicate in this section how to get over this difficulty.

Let $S$ be a Riemann surface. Let $\mu \geq 0$ be a Radon measure defined on an open set $\omega$ in S. Then, using an approximation theorem of Pfluger [9, page 192], we can show that there exists a superhamonic function $s$ on $\omega$ with associated measure $\mu$ in a local Riesz representation as explained in Lemma 3.3 (see [3, Theorem 2.3]). Let us symbolically denote this relation between $s$ and $\mu$ by $L s=-\mu$ on $\omega$.

Let now $d \sigma$ denote the surface measure on $S$. Then, given any locally $d \sigma$-integrable function $f$ on an open set $\omega$, let $\lambda$ be the signed measure on $\omega$ defined by $d \lambda=f d \sigma$. Construct as above two superharmonic functions $s_{1}$ and $s_{2}$ on $\omega$, such that $L s_{1}=-\lambda^{+}$and $L s_{2}=-\lambda^{-}$. Let us denote this relation between the $\delta$-superharmonic function $s=s_{1}-s_{2}$ and the locally $d \sigma$-integrable function $f$ by $L s=-f$.

We will say that $s=\left(s_{m}, s_{m-1}, \ldots, s_{1}\right)$ is a polyharmonic-superharmonic function of order $m$ in an open set $\omega$, if $s_{1}$ is superharmonic on $\omega$ and $L s_{i}=-s_{i-1}$ for $2 \leq i \leq m$. We will say that $s \geq 0$ if each $s_{i} \geq 0$. If there exists a polyharmonic-superharmonic function $s=\left(s_{m}, s_{m-1}, \ldots, s_{1}\right) \geq 0, s_{i} \neq \equiv$ for any $i$, on a domain $\Omega$ in $S$, we say that $\Omega$ is an $m$ harmonic Green domain in $S$.

Let now $\Omega$ be a Green domain in a Riemann surface $S$. As before, let $\bar{\Omega}$ be the Martin compactfication of $\Omega$, let $\Delta=\bar{\Omega} \backslash \Omega$ be the Martin boundary, and let $\Delta_{1}$ be the minimal boundary. Then, with the notations as in Section 4, we can prove the following.

Theorem 5.1. Let $\Omega$ be an m-harmonic Green domain in a Riemann surface S. Let $m \geq 1$ be an integer. Then, the following are equivalent.

(a) $s=\left(s_{m}, s_{m-1}, \ldots, s_{1}\right) \geq 0$ is a polyharmonic-superharmonic function of order $m$ in $\Omega$.

(b) For any $j, 1 \leq j \leq m$, there exist unique measures $\mu \in \pi_{j}$ and $v_{i} \in \wedge_{i}$ for $0 \leq i \leq$ $j-1$ such that

$$
s_{j}(x)=\int_{\Omega} G^{j}(x, y) d \mu(y)+\sum_{i=0}^{j-1} \int_{\Delta_{1}} k_{i}(X, x) d v_{i}(X) \quad \text { a.e. on } \Omega \text {. }
$$

(c) The above property (b) is satisfied for $j=m$. 
Proof. (a) $\Rightarrow$ (b). Fix $j, 1 \leq j \leq m$. Then $\left(s_{j}, s_{j-1}, \ldots, s_{1}\right)$ is a $j$-superharmonic function on $\Omega$, since $(-L) s_{i+1}=s_{i}$ for $1 \leq i \leq j-1$ and $s_{1}$ is superharmonic. Moreover, since $(-L) s_{i+1}$ $\geq 0$, each $s_{i}$ is a positive superharmonic function. Write $s_{1}=p_{1}+h_{1}$ as the unique sum of a potential $p_{1}$ and a positive harmonic function $h_{1}$. Let $(-L) p_{1}^{*}=p_{1}$ and $(-L) h_{1}^{*}=h_{1}$. Then $p_{1}^{*}$ and $h_{1}^{*}$ are superharmonic on $\Omega$ and

$$
(-L) s_{2}=p_{1}+h_{1}=(-L) p_{1}^{*}+(-L) h_{1}^{*}
$$

That is, $s_{2}=p_{1}^{*}+h_{1}^{*}+$ (a harmonic function) on $\Omega$. Since $s_{2} \geq 0, p_{1}^{*}$ has a subharmonic minorant on $\Omega$ and hence $p_{1}^{*}=\left(\right.$ a potential $\left.p_{2}\right)+$ (the greatest harmonic minorant of $p_{1}^{*}$, which may not necessarily be positive).

Then $s_{2}=p_{2}+u_{2}$, where $u_{2}$ is superharmonic on $\Omega$. Since $s_{2} \geq 0, p_{2} \geq-u_{2}$. Since $p_{2}$ is a potential and $-u_{2}$ is subharmonic, $-u_{2} \leq 0$. Hence $s_{2}=p_{2}+u_{2}$, where $p_{2}$ is a potential on $\Omega$ such that $(-L) p_{2}=p_{1}$ and $u_{2} \geq 0$ is superharmonic such that $(-L) u_{2}=h_{1}$.

Thus proceeding, we can write

$$
\left(s_{j}, \ldots, s_{2}, s_{1}\right)=\left(p_{j}, \ldots, p_{2}, p_{1}\right)+\left(u_{j}, \ldots, u_{2}, h_{1}\right)
$$

where $(-L) p_{i+1}=p_{i}$ for $1 \leq i \leq j-1$, and $p_{1}, \ldots, p_{j}$ are all potentials; $(-L) u_{i+1}=u_{i}$ for $2 \leq i \leq j-1$ and $(-L) u_{2}=h_{1}$.

Now take $\left(u_{j}, \ldots, u_{2}, h_{1}\right)$ and proceed as before. Note now $h_{1}$ is positive harmonic, so that we can write

$$
\left(u_{j}, \ldots, u_{2}, h_{1}\right)=\left(q_{j}, \ldots, q_{2}, h_{1}\right)+\left(f_{j}, \ldots, f_{3}, h_{2}, 0\right),
$$

where $(-L) q_{i+1}=q_{i}$ for $2 \leq i \leq j-1,(-L) q_{2}=h_{1}$, and each $q_{i}$ is a potential; $(-L) f_{i+1}=$ $f_{i} \geq 0$ for $3 \leq i \leq j-1,(-L) f_{3}=h_{2}$, and $(-L) h_{2}=0$, so that $h_{2}$ is positive harmonic.

Then take $\left(f_{j}, \ldots, f_{3}, h_{2}, 0\right)$ and follow the same procedure, so that

$$
\left(f_{j}, \ldots, f_{3}, h_{2}, 0\right)=\left(r_{j}, \ldots, r_{3}, h_{2}, 0\right)+\left(g_{j}, \ldots, g_{4}, h_{3}, 0,0\right),
$$

where $(-L) r_{i+1}=r_{i}$ for $3 \leq i \leq j-1,(-L) r_{3}=h_{2}$ and each $r_{i}$ is a potential; $(-L) g_{i+1}=$ $g_{i} \geq 0$ for $4 \leq i \leq j-1,(-L) g_{4}=h_{3}$ and $(-L) h_{3}=0$, so that $h_{3}$ is harmonic $\geq 0$.

Thus proceeding, we finally arrive at the decomposition

$$
\left(s_{j}, \ldots, s_{1}\right)=\left(p_{j}, \ldots, p_{1}\right)+\left(q_{j}, \ldots, q_{2}, h_{1}\right)+\left(r_{j}, \ldots, r_{3}, h_{2}, 0\right)+\cdots+\left(h_{j}, 0, \ldots, 0\right) .
$$

Let $(-L) p_{1}=\mu$; let $v_{i}(1 \leq i \leq j)$ be the positive Radon measure on $\Delta$ with support in $\Delta_{1}$, associated with the positive harmonic function $h_{i}$ in the Martin representation.

Then $s_{j}=p_{j}+q_{j}+r_{j}+\cdots+h_{j}$ has the integral representation

$$
s_{j}(x)=\int_{\Omega} G^{j}(x, y) d \mu(y)+\sum_{i=0}^{j-1} \int_{\Delta_{1}} k_{i}(X, x) d v_{i}(X) \quad \text { a.e. on } \Omega \text {. }
$$

(b) $\Rightarrow(\mathrm{c}) . j=m$ is a particular case of (b). 
(c) $\Rightarrow(\mathrm{a})$. By the assumption,

$$
s_{m}(x)=\int_{\Omega} G^{m}(x, y) d \mu(y)+\sum_{i=0}^{m-1} \int_{\Delta_{1}} k_{i}(X, y) d v_{i}(X) \quad \text { a.e. }
$$

Hence we can express $s_{m}$ in the form $s_{m}(x)=p_{m}(x)+\sum_{j=0}^{m-1} q_{j}(x)$. We can calculate to find that $(-L)^{i} p_{m}$ is a potential for $1 \leq i \leq m-1$ and $(-L)^{m} p_{m}=\mu$, a positive Radon measure; and $(-L)^{i} q_{j}$ is a potential for $1 \leq i \leq j-1$ and $(-L)^{j} q_{j}=0$.

Write now $(-L) s_{m}=s_{m-1},(-L) s_{m-1}=s_{m-2}, \ldots,(-L) s_{2}=s_{1}$. We can see that each $s_{i}$ $(1 \leq i \leq m)$ is a positive superharmonic function and $(-L) s_{i+1}=s_{i}$ for $1 \leq i \leq m-1$.

Hence $s=\left(s_{m}, s_{m-1}, \ldots, s_{1}\right) \geq 0$ is a polyharmonic-superharmonic function of order $m$.

\section{References}

[1] A. Abkar and H. Hedenmalm, A Riesz representation formula for super-biharmonic functions, Annales Academiæ Scientiarium Fennicæ Mathematica 26 (2001), no. 2, 305-324.

[2] V. Anandam, Biharmonic Green functions in a Riemannian manifold, Arab Journal of Mathematical Sciences 4 (1998), no. 1, 39-45.

[3] __ Biharmonic classification of harmonic spaces, Revue Roumaine de Mathématiques Pures et Appliquées 45 (2000), no. 3, 383-395.

[4] S. Axler, P. Bourdon, and W. Ramey, Harmonic Function Theory, Graduate Texts in Mathematics, vol. 137, Springer, New York, 1992.

[5] T. Bagby and P. Blanchet, Uniform harmonic approximation on Riemannian manifolds, Journal d'Analyse Mathématique 62 (1994), 47-76.

[6] M. Brelot, Fonctions sousharmoniques associées à une mesure, Studii şi Cercetari Matematice, Iaşi, Academiei Române, Section Jasy, Roumania 2 (1951), 114-118.

[7] __ Axiomatique des fonctions harmoniques, Les Presses de l'Université de Montréal, Montréal, 1966.

[8] _ On Topologies and Boundaries in Potential Theory, Lecture Notes in Mathematics, vol. 175, Springer, Berlin, 1971.

[9] A. Pfluger, Theorie der Riemannschen Flächen, Springer, Berlin, 1957.

[10] L. Sario, A criterion for the existence of biharmonic Green's functions, Journal of the Australian Mathematical Society, Series A 21 (1976), no. 2, 155-165.

V. Anandam: Department of Mathematics, King Saud University, P.O. Box 2455,

Riyadh 11451, Saudi Arabia

E-mail address: vanandam@ksu.edu.sa

S. I. Othman: Department of Mathematics, King Saud University, P.O. Box 2455,

Riyadh 11451, Saudi Arabia

E-mail address: sadoon@ksu.edu.sa 


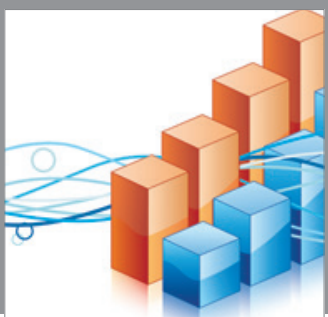

Advances in

Operations Research

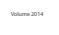

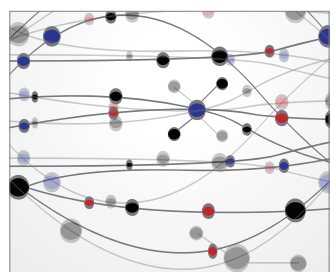

\section{The Scientific} World Journal
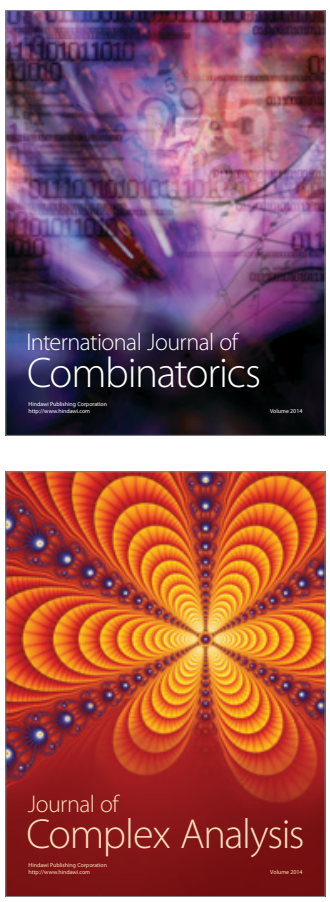

International Journal of

Mathematics and

Mathematical

Sciences
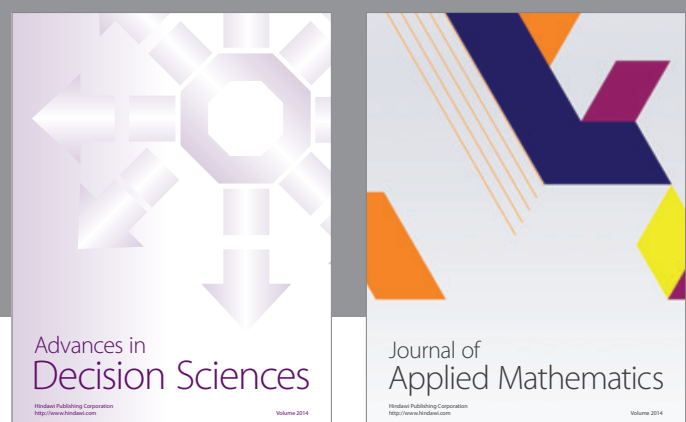

Journal of

Applied Mathematics
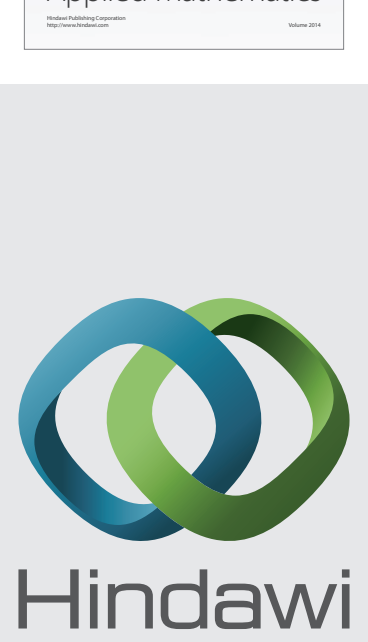

Submit your manuscripts at http://www.hindawi.com
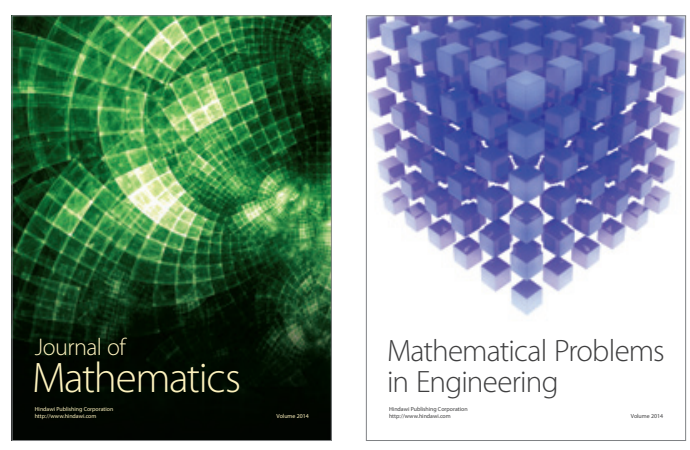

Mathematical Problems in Engineering
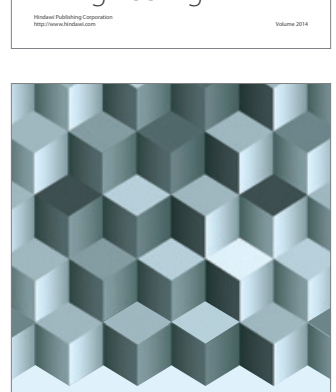

Journal of

Function Spaces
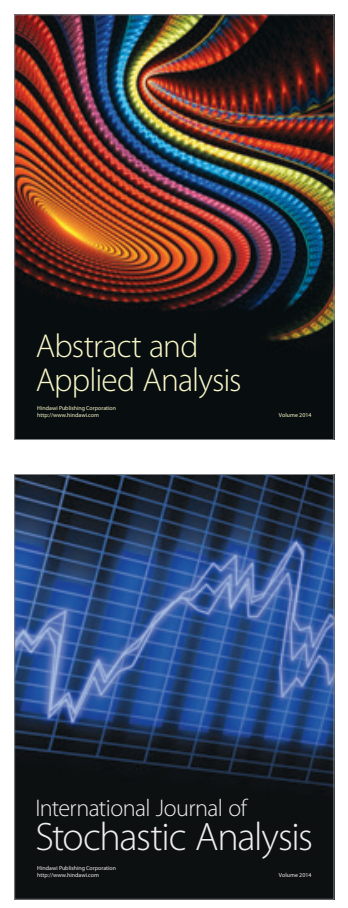

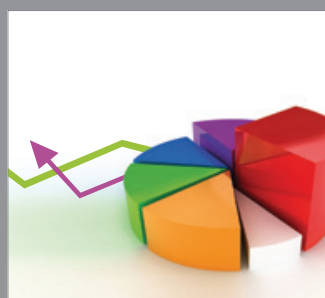

ournal of

Probability and Statistics

Promensencen
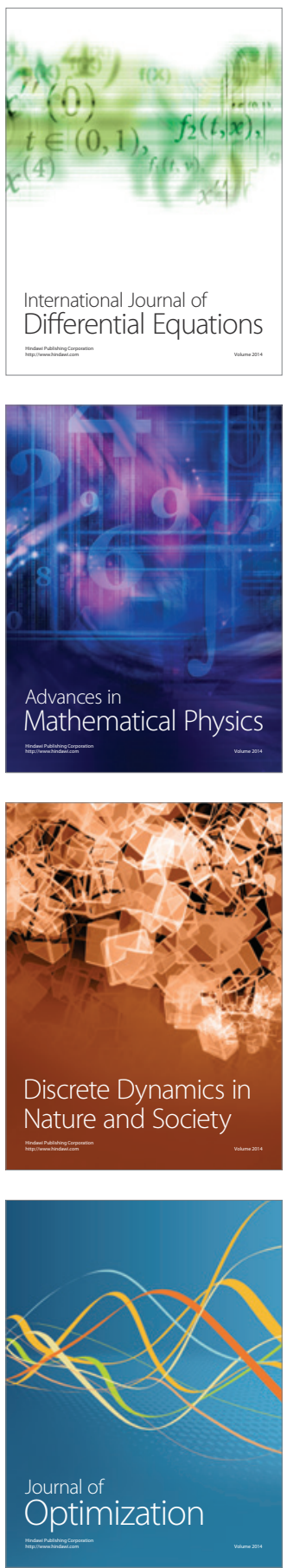